\title{
Respiratory viral infections by Non-influenza viruses are associated with more adverse clinical outcome in patients with underlying liver disease: a single centre laboratory based study.
}

\author{
Ekta Gupta ${ }^{1}$, Abhishek Padhi ${ }^{1}$, Kavita Aggarwal ${ }^{2}$, Krithiga Ramachandran ${ }^{2}$, Reshu \\ Agarwal $^{2}$, Samba Siva Pasupulleti ${ }^{2}$, Debajyoti Bhattacharya ${ }^{2}$, Rakhi Maiwall ${ }^{2}$, and Shiv \\ $\operatorname{Sarin}^{2}$ \\ ${ }^{1}$ Institute of Liver and Biliary Sciences \\ ${ }^{2}$ ILBS
}

August 7, 2020

\begin{abstract}
Background Respiratory viral infections are an important cause of acute respiratory tract infections. They are caused by both Influenza and non influenza viruses. Respiratory viral infections are known to be associated with severe clinical outcome especially in the critically ill. A constant surveillance is needed for early etiological identification which can help in timely and appropriate management. Methods In this retrospective study, clinical records of all adult liver disease patients with clinically confirmed ARI, whose request for respiratory viral testing were received in the virology laboratory during September 2016 - March 2019 were reviewed. Respiratory viruses were identified by real time PCR on FilmArray 2.0 instrument (BioFire Diagnostics, Utah, USA) using Respiratory panel as per the manufacturer's instructions. Results Of the 603 patients of liver disease with clinically confirmed influenza like illness, over all incidence of respiratory viral infection was $24.3 \%$ ( $\mathrm{n}=147$ ). Infections by non-influenza viruses $(87,59.1 \%)$ were more than influenza group of viruses. Mortality was higher in non influenza group $(43,49.4 \%)$ as compared to influenza $(24,40 \%)[\mathrm{p}=0.015]$ being maximum in Rhinovirus, 22 (32.8\%). Two peaks were observed in both influenza and non influenza groups, first in the months of January and February and the other one in August and October. Conclusion With the emergence of SARS- CoV-2 it has now become imperative for a constant surveillance of the non influenza viruses for early etiological identification of the respiratory viral infection for proper and timely management in the critically ill.
\end{abstract}

\section{Hosted file}

RVI WILEY.docx available at https://authorea.com/users/349432/articles/474448-respiratoryviral-infections-by-non-influenza-viruses-are-associated-with-more-adverse-clinicaloutcome-in-patients-with-underlying-liver-disease-a-single-centre-laboratory-based-study 